\title{
Automated Mental Stress Recognition through Mobile Thermal Imaging
}

\author{
Youngjun Cho \\ UCL Interaction Centre, Faculty of Brain Sciences \\ University College London \\ London, United Kingdom
}

\begin{abstract}
Mental stress is a critical problem in our modern society. This form of stress strongly affects our well being, and technology is needed to help us to manage health problems. The ability to automatically recognize a person's mental stress can be fundamental in supporting stress and health management. This research focuses on the use of mobile thermal imaging, a new and less explored sensor, to merge the measurement of multiple physiological signatures into one sensor and to build a reliable mental stress automatic recognition model. Mobile thermal imaging has greater potentials for real-world applications given that it is small and light weight, and requires low computation cost. To make mobile thermal imaging a robust multimodal stress sensor, we have so far contributed: i) a new robust respiration tracking method; and ii) a novel respiration-based automatic stress recognition model that works in ubiquitous settings. We are currently investigating new thermal signatures from underexplored body regions (i.e. trapezius muscle) and formulating a research framework to fuse multiple thermal signatures for more reliable stress recognition outcomes.
\end{abstract}

\section{Introduction}

With technology becoming pervasive in our everyday life, the ability to recognize human psychophysiological states has become fundamental in human computer interaction. Studies show that mental stress, a common problem affecting our life in this modern society, could be automatically tracked by using those sensing technologies, in turn opening the way to new stress management support strategies (e.g., [1], [2]). As mental stress is a complex phenomenon which influences a person's neural and physiological processes, various approaches have been proposed to extract signatures of stress responses. Sensing channels for cardiac activity (e.g. heart rate, heart rate variability, blood pressure), respiratory activity (e.g. breathing rate, tidal volume), temperature, skin conductance (e.g. level and response) and neural activity (EEG) have been employed for physiological measures of stress (e.g. [1]-[5]). Most of these channels require sensor contact.

Interestingly, similarly to behavioural signatures which can be collected without sensor contact (e.g. body motion in [6], voice in [7]), researchers have shown that affect-related physiological signatures (e.g. temperature, cardiac and respiratory activations) can also be captured via non-contact measurements through thermal imaging (e.g. respiration monitoring in [8]) and RGB cameras (i.e. remote photoplethysmography (PPG) in [9]). Whilst RGB camerabased methods raise illumination and privacy issues, thermography is much less affected by those constraints [10]. In addition, studies have shown that different types of autonomic nervous system activities can be simultaneously read through the use of thermal imaging (e.g. temperature direction in [4], respiration in [8], heart rate in [11]).

Although earlier approaches provided initial evidences of the relation between affect and temperature, a variety of body regions and their thermal signatures have been much less investigated than thermal directionality of facial and palm regions [4], [12]-[17]. Little is indeed known about how such areas respond to stress level. Furthermore, all of the earlier works have employed very heavy and expensive thermographic systems, which cannot be easily set up in certain positions and are less likely to be used in $\mathrm{HCI}$ applications (its cost range: over $£ 1,000 \sim$ approximately $£ 10,000$ in 2017).

More recently, advanced thermography technology has emerged, producing a new category of thermographic systems: mobile, low-cost thermal imaging. Despite its relatively low quality thermal imaging outputs, this technology could help bridge the gap between the theoretical findings from the lab environment and real world applications in the wild. Indeed, its portability (e.g. small size and low computational resource requirement) allows it to be easily attached not just to mobile phones but also be integrated into our clothes and accessories. Extending the body of earlier work on thermal imaging and affect recognition, this research aims to explore how to bring Mobile Thermography into real world human computer interaction as a multimodal mental stress sensor.

\section{Literature Review: A Short Summary}

Thermal imaging is a key non-invasive method to study heat distributions in materials and organisms based on the interpretation of electromagnetic radiation [10]. Since the human cutaneous and subcutaneous skin releases infrared radiations, they can be remotely interpreted and transformed into thermograms [18]. Indeed, thermal imaging has been widely used in medical applications as a diagnostic tool, such as for the identification of tumours and inflammatory diseases [19]. With advancements in commercial thermal imaging technologies, thermography has begun to extend its application context to the analysis of people's physiological processes and affective states. Indeed, it has been shown that physiological 
signatures, such as thermal directional changes (e.g., [4], [12][14]), cardiac (e.g., [11]), perspiratory (e.g., [15]) and respiratory (e.g., [8]) indices, and muscular activations related to facial expressions (e.g. FACS decoding [20]), can be captured through thermal imaging. Researchers have investigated thermal signatures of one's face in relation to mental stress, (e.g., [4], [13], [21]-[23]). For example, it was found that a person's nose tip could show temperature drops under cognitive load inducing mental stress [4]. However, the results on what such thermal change should be, including its direction, are not clear. All these works have been focussing primarily on facial regions and have used high performance, high-end, heavy thermal imaging systems. Hence, whilst it is evident that thermal imaging has greater potentials for automatic stress recognition, its applicability to real-life ubiquitous everyday settings and needs remains to be investigated.

\section{Methodology: An Overview}

Methodology proposed for this research is organised in three stages: biomedical thermal image processing for automatically tracking ROIs; physiology measurements in ubiquitous settings; and automated mental stress recognition based on both uni- and multi-modal models as illustrated in Figure 1. The thermal image processing stage aims to develop new methods to overcome the challenges posed by low cost mobile thermal imaging including going beyond controlled static laboratory settings. The physiology measurements stage aims at proposing new physiological signatures that facilitate the capturing of the relationship between stress and respiratory, cardiac, eye blinks and skeletal muscle activations. In particular, we aim to identify thermal signatures that can be reliably extracted during everyday tasks with dynamic temperature environments. In addition, we aim to explore muscle activation over the upper trapezius muscle, an underexplored body area in relation to physical and mental stress. Lastly, the final stage focuses on building models for automated stress recognition based on either (respiration, blood volume pulse, skeletal muscle activation-based) unimodal signals, and a multimodal fusion of those signals. For this stage, this research will explore and compare modelling approaches to stress recognition. When necessary, data augmentation techniques will be explored and proposed to use machine learning techniques that require a large dataset. Finally, the work will contribute a dataset of thermal data in both constrained (for comparison with state of the art approaches) and everyday settings to evaluate performance in the wild.

\section{Physical Interface Concepts for Mobile Thermal Imaging}

Among low-cost, mobile thermal imaging systems commercially available, FLIR One, which was the cheapest one in Jan. 2017, is chosen as the mobile thermal imaging sensor in this research. To support unconstrained situations, the camera will be integrated with devices already used: rigid (e.g., PC, desk) or non-rigid (e.g., headset with microphone and Augmented Reality interface like HoloLens) structures to

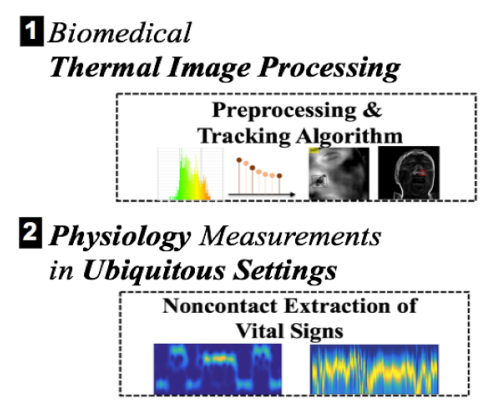

3 Automated Mental Stress Recognition

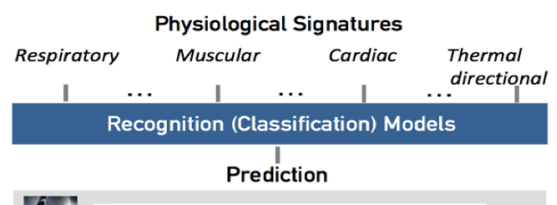

I guess you've felt stressed out

Figure. 1. Proposed methodology: biomedical thermal image processing for the automated ROI tracking - physiology measurements - automated stress recognition.

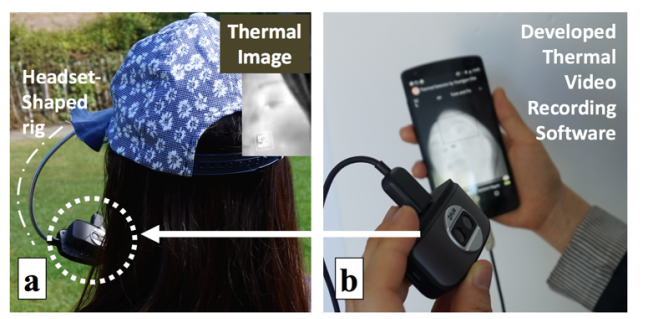

Figure. 2. Physical interface concepts for mobile thermal imaging: Monitoring thermal dynamics over a person's upper body with the aid of (a) a hardware steel frame (e.g. a headset interface attached to a cap for the camera viewing a person's face) for mobile setting, and (b) without the aid of hardware, i.e. a handheld concept.

unobtrusively monitor a person's mental stress. For the purpose of this research, we have built some of these physical structures as shown in Figure 2. To monitor a person's thermal signatures while walking and moving, a hardware frame (e.g. a headsetshaped interface) (see Figure 2a) can be used to place the thermal device near a person's upper body, similar to set-ups used in other studies [24], [25]. The portable mount-setup can help the thermographic camera to effectively focus on facial and upper trapezius regions, while at the same time they may not interfere too much a person's view. As an alternative setup, Figure $2 \mathrm{~b}$ describes a handheld concept. In comparison with the hardware-aided concept, this handheld design provides more flexibility and scalability for recovering physiological signatures and recognising the mental stress. Users can then use the system ad-hoc in situations when they need to monitor their condition. This flexible, handheld setup enables many other use cases, such as attaching the system on a desktop, desk or handle of the bicycle, at a dashboard in the car. 


\section{Contributions made so far}

This section introduces some of key works done or ongoing for the automated mental stress recognition using mobile thermal imaging.

\subsection{Stage 1: Optimal Quantisation for Automated ROI Tracking in Mobile Settings}

Visual motion-tracking algorithms in computer vision can be used for automatically tracking a ROI on thermal videos (e.g. [26] used in [8]). However, there is a problematic challenge when those vision algorithms are applied to thermal imaging sequences collected from real-world environment and mobile settings, i.e., beyond lab studies. Thermal dynamics (i.e. different ambient temperature and its dynamic variations) lead to tracking imperfections since they are likely to change the morphological properties of the body appearance in the thermogram. For that reason, we proposed a novel Optimal Quantisation method reported in [27]. Earlier works generally used a fixed temperature range of interest to convert a temperature matrix to an image (i.e., quantisation [28]), vulnerable to the environmental thermal dynamics. On the other hand, our proposed method handles those factors by searching the optimal thermal range of interest on every single frame reflecting time-varying thermal dynamics, contributing to produce highly reliable performance in automated ROI tracking. For more technical details, we refer to [27].

\subsection{Stage 2: Physiology Measurements Through Mobile Thermal Imaging}

1) Robust Respiration Tracking in ubiquitous settings

Researchers have shown that respiratory patterns can be computed from the thermal changes around the nostril (e.g., [8]). Respiratory thermal signature is interesting because it could be more informative over time in comparison with other types of known signatures (e.g. thermal directionality is simply a discrete value) and mental stress affects its irregularity, but has not been employed for detecting stress in thermography-based earlier works. Accordingly, we proposed a new respiration tracking method in [27] so as to reliably recover respiratory patterns using mobile thermal imaging. While controlled static contexts have been mainly targeted in the body of work (e.g., [8]), our proposed method supports real-world situations beyond constrained indoor-laboratory settings. In [27], key challenges were identified for the use of mobile thermal imaging to track one's respiratory signals in unconstrained settings: a) ROI tracking errors due to motion artefacts and b) low respiratory signal quality due to the low spatial resolution of the imaging and to mobile situations. To overcome such issues, we proposed the Thermal Gradient Flow and Thermal Voxel Integration algorithms in [27]. The former technique is mainly based on building thermal-gradient magnitude maps for enhancing the boundary around the nostril regions, which in turn contribute to making the system robust to motion artefacts. The latter one projects a $2 \mathrm{D}$ thermal matrix onto a $3 \mathrm{D}$ space by taking a unit thermal element as a thermal voxel. This method results in producing higher quality of breathing patterns. The proposed methods were thoroughly evaluated in three studies which were conducted with different levels of challenges producing strong correlation ( $\mathrm{r}=0.9983)$ with the ground truth signals (i.e., breathing-belt).

\section{2) Heart Rate Monitoring}

Cardiac activation indices could be recovered from subtle thermal fluctuations around superficial blood vessels since the temperature is affected by the blood flow [11]. Vessels on neck or forehead areas, which can be clearly seen on thermograms, were mainly explored in previous studies (e.g. [11]). However, since these areas are often occluded by clothes or hairs in real life situations, a different processing strategy is necessary. In one of our ongoing studies, we are investigating how to remove processes for the registration and localization of artery vessels and how to recover blood dispersions of facial capillary networks as shown in Figure 3. Again, the proposed Optimal Quantisation and Thermal Gradient Flow techniques [27] could help reliably extract local thermal sequences in relation to capillary systems (see Figure 3b).

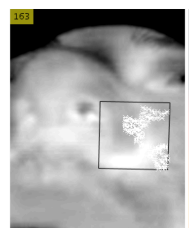

(a)

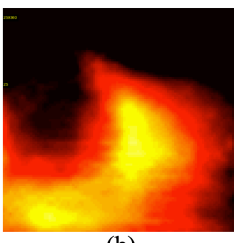

(b)

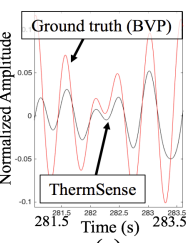

(c)
Figure 3. (a) Thermal observation of capillary networks, (b) extraction of thermal dispersions from a tracked ROI and (c) cardiac pulse signals recovered by our very preliminary approach.
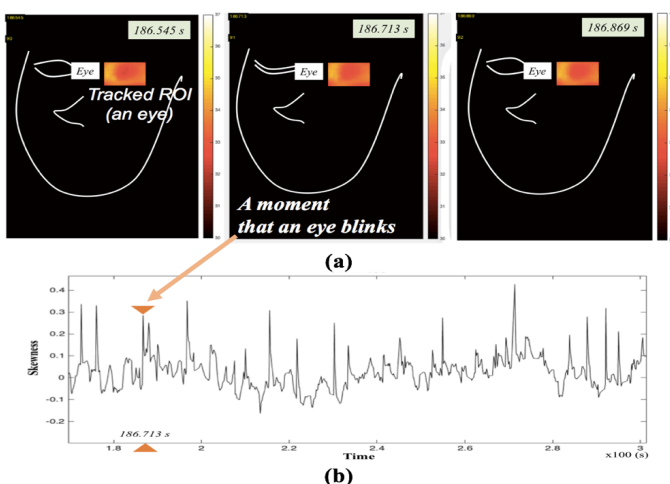

Figure 4. Extraction of Eye blinks: (a) the eye-ROI on thermal image sequences during the eye-blink moment, (b) skewness along with time.

\section{3) Extraction of Eye Blinks}

Despite thermal signatures of eye blinks being underexplored, it is also one of important physiological cues to a person's psychological states [29]. But, it is difficult to extract one's eye-blink pattern through low-resolution mobile thermal imaging since thermal representation of one's eye is likely to get blurred. For the recognition of minute thermal changes on the eye-ROI by eye blinks, we are exploring the use of Skewness in one of our ongoing works. It can assist to sense a moment when an eye is blinking as can be seen in Figure 4. Figure 4a shows the eye-ROI on thermal image sequences during the eye blink moments (collected at 186.545s, 186.713s and 186.869s) and 
local peaks of Skewness is matched with the blinks as shown in Figure $4 b$.

\section{4) Skeletal Muscle Activation: A New Thermal Signature from Underexplored ROIs}

This research aims to explore new underexplored regions which could provide a strong thermal signature in relation to a person's psychological stress. Mobile thermal imaging can be a crucial tool for monitoring body areas given the advantage of its light weight. A mobile light thermal camera can be set up near a person's body in a way that it was not possible before with high-end expensive and heavy thermal cameras. Using this setup, this research attempts to identify informative thermal signatures from skeletal muscles (e.g., trapezius) in relation to the muscle activation under cognitive load. In one of our ongoing studies, a variety of statistical features have been extracted from one's upper trapezius as illustrated in Figure 5.

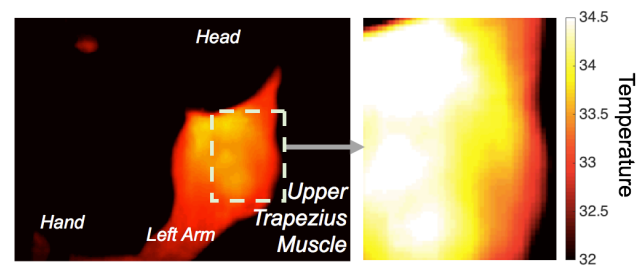

Figure 5. A thermal image over a person's upper body (top view): to identify thermal signatures from the upper trapezius muscle.

\subsection{Stage 3: Unimodal Stress Recognition by going deeper into breathing dynamics}

The main goal of this research is to build a reliable automatic stress recognition system which can support unconstrained real-world settings. Toward this goal, we proposed DeepBreath, a new respiration-based unimodal stress detection model, in one of our previous works [30]. The robust respiration tracking method discussed in Section 4.2.1 is fundamentally used to extract the breathing pattern. In earlier studies on mental stress recognition, gross statistical features (e.g, mean) of respiration patterns have been generally used together with those from other physiological ones (e.g. [1]). However, in these works, the contribution of gross respiratory features to automated stress recognition was not clear (e.g. different results in [2] and [31]). In [30], a novel 2D representation (signature) of breathing pattern dynamics (i.e., two dimensional respiration variability spectrogram, RVS) was proposed, inspired by the fact that psychological stress affects the regularity of a person's breathing pattern [32]. This new form of input signature condenses dynamic respiratory information. Features regarding respiratory irregularity can be learnt by a deep learning framework. In [30], the convolutional neural network $(\mathrm{CNN})$ architecture was employed for feature learning. Finally, a data augmentation technique was designed to activate the deep network with a small-scale dataset. Two types of standard stress induction tasks (i.e., Stroop Colour Word Test and Arithmetic Test) were used to collect the RVS datasets and to evaluate the proposed stress recognition system.
The study results showed that DeepBreath produced above chance level accuracies in discriminating multiple stress levels.

\section{Future Works: Multimodal Fusion and Intervention}

This research plans to fuse different thermal signatures for achieving highly robust performances of the psychological stress recognition. The 2D input signature of respiration variability in [30] and thermal signatures from newly explored ROIs (i.e. skeletal muscles) will be merged together with known thermal signatures such as thermal directional changes on facial areas. Different learning models including deep learning model proposed in [30] and dynamic Bayesian network (e.g., hidden Markov) based ones (to handle timevarying thermal signatures) will be investigated and possibly merged into one decision model. We are also exploring the use of co-learning methods for this purpose [33]. Finally, the proposed multimodal fusion methods will be evaluated with an ad-hoc built basic proof-of-concept coaching system. For this final phase, multimodal feedback (e.g. tactile and auditory feedback [34], [35]) along with stress predictions can be used to raise a person's stress awareness.

The datasets of labelled thermal images and extracted physiological patterns will be incrementally made as results are published (available at http://youngjuncho.com/datasets/).

\section{Conclusion}

Toward building a robust stress recognition system, this research proposes thermal image processing methods for automatic ROI tracking through thermal imaging (can be applicable to any types of thermal cameras), methods for physiology measurements, and machine learning models for automatically discriminating a person's mental stress levels. To overcome key challenges identified from the literature (e.g. the automated ROI tracking issues, impracticality of the expensive high-precision thermal camera, limitations of basic thermal signatures in automatically recognising a person's stress), this research focuses on the use of mobile thermal imaging in reallife like situations. In detail, a new quantisation method was investigated. A robust respiration tracking method, which works in ubiquitous settings, was proposed. In addition, we proposed a novel respiration-based unimodal stress recognition system which produces high accuracy results. In order to build a multi-modal fusion model to achieve more reliable recognition performances, we have started to explore how to extract other types of physiological signatures through mobile thermal imaging. we believe this research could open a new era of thermal imaging-based research on a person's affective states.

\section{Acknowledgments}

This research is conducted under the supervision of Prof. Nadia Bianchi-Berthouze (Primary Supervisor), Dr. Simon Julier and Dr. Nicolai Marquardt (Secondary Supervisors), and supported by University College London Overseas Research Scholarship awarded to top quality international postgraduate 
students.

\section{References}

[1] J. A. Healey and R. W. Picard, "Detecting stress during real-world driving tasks using physiological sensors", IEEE Trans. Intell. Transp. Syst., vol. 6, no. 2, pp. 156-166, 2005.

[2] J.-H. Hong, J. Ramos, and A. K. Dey, "Understanding Physiological Responses to Stressors During Physical Activity", in Proceedings of the 2012 ACM Conference on Ubiquitous Computing, 2012, pp. 270-279.

[3] S. A. Hosseini and M. A. Khalilzadeh, "Emotional Stress Recognition System Using EEG and Psychophysiological Signals: Using New Labelling Process of EEG Signals in Emotional Stress State", in 2010 International Conference on Biomedical Engineering and Computer Science, 2010, pp. 1-6.

[4] V. Engert, A. Merla, J. A. Grant, D. Cardone, A. Tusche, and T. Singer, "Exploring the Use of Thermal Infrared Imaging in Human Stress Research", PLOS ONE, vol. 9, no. 3, p. e90782, 2014

[5] J. Hernandez, R. R. Morris, and R. W. Picard, "Call Center Stress Recognition with Person-Specific Models", in Affective Computing and Intelligent Interaction, 2011, pp. 125-134.

[6] A. Kleinsmith and N. Bianchi-Berthouze, "Affective Body Expression Perception and Recognition: A Survey", IEEE Trans. Affect. Comput., vol. 4, no. 1, pp. 15-33, 2013.

[7] N. D. Lane, P. Georgiev, and L. Qendro, "DeepEar: Robust Smartphone Audio Sensing in Unconstrained Acoustic Environments Using Deep Learning", in Proceedings of the 2015 ACM International Joint Conference on Pervasive and Ubiquitous Computing, 2015, pp. 283-294.

[8] C. B. Pereira, X. Yu, M. Czaplik, R. Rossaint, V. Blazek, and S. Leonhardt, "Remote monitoring of breathing dynamics using infrared thermography", Biomed. Opt. Express, vol. 6, no. 11, pp. 4378-4394, 2015.

[9] W. Verkruysse, L. O. Svaasand, and J. S. Nelson, "Remote plethysmographic imaging using ambient light. ”, Opt. Express, vol. 16, no. 26, pp. 21434-21445, 2008.

[10] J. M. Lloyd, Thermal Imaging Systems. Springer Science \& Business Media, 2013

[11] M. Garbey, N. Sun, A. Merla, and I. Pavlidis, "Contact-Free Measurement of Cardiac Pulse Based on the Analysis of Thermal Imagery", IEEE Trans. Biomed. Eng., vol. 54, no. 8, pp. 1418 1426,2007

[12] L. Gane, S. Power, A. Kushki, and T. Chau, "Thermal Imaging of the Periorbital Regions during the Presentation of an Auditory Startle Stimulus", PLOS ONE, vol. 6, no. 11, p. e27268, 2011.

[13] H. Genno et al., "Using facial skin temperature to objectively evaluate sensations", Int. J. Ind. Ergon., vol. 19, no. 2, pp. 161171, 1997.

[14] I. Pavlidis, N. L. Eberhardt, and J. A. Levine, "Human behaviour: Seeing through the face of deception", Nature, vol. 415 , no. 6867 , pp. 35-35, 2002.

[15] I. Pavlidis et al., "Fast by Nature - How Stress Patterns Define Human Experience and Performance in Dexterous Tasks", Sci. Rep., vol. 2, 2012.

[16] B. R. Nhan and T. Chau, "Classifying Affective States Using Thermal Infrared Imaging of the Human Face", IEEE Trans. Biomed. Eng., vol. 57, no. 4, pp. 979-987, 2010.

[17] M. M. Khan, R. Ward, and M. Ingleby, "Toward Use of Facial Thermal Features in Dynamic Assessment of Affect and Arousal Level”, IEEE Trans. Affect. Comput., vol. PP, no. 99, pp. 1-1,
2016.

[18] I. Fujimas, "Pathophysiological expression and analysis of far infrared thermal images", IEEE Eng. Med. Biol. Mag., vol. 17, no. 4, pp. 34-42, 1998.

[19] E. F. J. Ring and K. Ammer, "Infrared thermal imaging in medicine", Physiol. Meas., vol. 33, no. 3, p. R33, 2012.

[20] S. Jarlier et al., "Thermal Analysis of Facial Muscles Contractions", IEEE Trans. Affect. Comput., vol. 2, no. 1, pp. 29, 2011.

[21] C. K. L. Or and V. G. Duffy, "Development of a facial skin temperature-based methodology for non-intrusive mental workload measurement", Occup. Ergon., vol. 7, no. 2, pp. 83-94, 2007.

[22] J. A. Veltman, W. K. Vos, and TNO Defensie en Veiligheid, "Facial temperature as a measure of mental workload", in International Symposium on Aviation Psychology, 2005, pp.597601

[23] C. Puri, L. Olson, I. Pavlidis, J. Levine, and J. Starren, "StressCam: Non-contact Measurement of Users' Emotional States Through Thermal Imaging”, in CHI '05 EA, 2005, pp. $1725-1728$

[24] C. Harrison, H. Benko, and A. D. Wilson, "OmniTouch: Wearable Multitouch Interaction Everywhere", in Proceedings of the 24th Annual ACM Symposium on User Interface Software and Technology, 2011, pp. 441-450.

[25] Y. Cho, S. Kim, and M. Joung, "Proximity Sensor and Control Method Thereof", US Patent, patent number: 9703368, 2017.

[26] X. Mei and H. Ling, "Robust Visual Tracking and Vehicle Classification via Sparse Representation", IEEE Trans. Pattern Anal. Mach. Intell., vol. 33, no. 11, pp. 2259-2272, 2011.

[27] Y. Cho, S. J. Julier, N. Marquardt, and N. Bianchi-Berthouze, "Robust tracking of respiratory rate in high-dynamic range scenes using mobile thermal imaging", Biomed. Opt. Express, 2017.

[28] P. Heckbert, "Color Image Quantization for Frame Buffer Display", in Proceedings of the 9th Annual Conference on Computer Graphics and Interactive Techniques, 1982, pp. $297-$ 307.

[29] P. P. Caffier, U. Erdmann, and P. Ullsperger, "Experimental evaluation of eye-blink parameters as a drowsiness measure", Eur. J. Appl. Physiol., vol. 89, no. 3-4, pp. 319-325, 2003.

[30] Y. Cho, N. Bianchi-Berthouze, and S. J. Julier, "DeepBreath: Deep Learning of Breathing Patterns for Automatic Stress Recognition using Low-Cost Thermal Imaging in Unconstrained Settings", in Affective Computing and Intelligent Interaction (ACII), 2017.

[31] Y. Masaoka and I. Homma, "Anxiety and respiratory patterns: their relationship during mental stress and physical load", Int. J. Psychophysiol., vol. 27, no. 2, pp. 153-159, 1997.

[32] P. Grossman, "Respiration, Stress, and Cardiovascular Function", Psychophysiology, vol. 20, no. 3, pp. 284-300, 1983.

[33] T. Baltrušaitis, C. Ahuja, and L.-P. Morency, "Multimodal Machine Learning: A Survey and Taxonomy", ArXiv170509406, 2017

[34] Y. Cho, A. Bianchi, N. Marquardt, and N. Bianchi-Berthouze, "RealPen: Providing Realism in Handwriting Tasks on Touch Surfaces Using Auditory-Tactile Feedback", in Proceedings of the 29th Annual Symposium on User Interface Software and Technology, 2016, pp. 195-205.

[35] D.-S. Kwon, T.-H. Yang, and Y.-J. Cho, "Mechatronics technology in mobile devices", Ind. Electron. Mag. IEEE, vol. 4, no. 2 , pp. $36-41,2010$. 